\title{
Efficient Algorithm for Varying Area based Shadow Detection in Video Sequences
}

\author{
Jasmin T Jose \\ Department of Computer \\ Science and Engineering, \\ National Institute of \\ Technology, Calicut.
}

\author{
V. K. Govindan \\ Department of Computer \\ Science and Engineering, \\ National Institute of \\ Technology, Calicut.
}

\begin{abstract}
Whenever the illumination from the light source is occluded by an object, shadow will be created in the video sequence. Shadow is a main factor affecting computer vision performance. Sometimes it will provide useful information about objects. However, the reliability of many vision algorithms may be reduced because of this same shadow. Therefore, to improve the performance of Computer vision processes, shadow detection and removal is an important preprocessing step. Moving Shadow detection has an important role in computer vision applications including video conference, vehicle tracking, and three-dimensional (3-D) object identification. This paper proposed a simple and efficient method to detect the moving shadow from video sequences. The basic principles underlying the area variation of the shadow from frame to frame and the relationship between the position of light source and the direction of object movement are employed in this approach. The performance of the technique is demonstrated and it is found to be efficient in detection and removal of shadows.
\end{abstract}

\section{General Terms}

Computer Vision, Shadow Detection.

\section{Keywords}

Computer Vision, Foreground Extraction, Background Subtraction.

\section{INTRODUCTION}

Shadow removal process can significantly improve and facilitate the performance of certain computer vision tasks. Because of the undesirable effect of Shadows on image analysis, much attention was paid to the area of shadow detection and removal, and covered many of the applications like traffic surveillance, face recognition and image segmentation. But shadow detection still remains an extremely challenging problem, particularly from a single image. Decomposition of a single image into a shadow part and a non-shadow part is a difficult problem.

It is important that the shadow areas are less illuminated than it's surroundings and the rate of change of intensity in shadow area is lesser than that in its surroundings. Generally, shadow is divided into two types, first, the Self shadow, the object part that does not receive proper light from light source. Second, Cast shadow or moving cast shadow (particularly for video images), the projection of the moving object on the background due to the light [1].

Cast shadow can be further divided into umbra and penumbra region. The umbra region is the part where direct light is completely blocked and the penumbra region is the part where light is partially blocked. Because of different properties of the cast and self shadows, algorithms to handle these two kinds of shadows are also different.

In computer vision algorithms like image segmentation, object detection and video surveillance, moving shadow detection has an important role. Shadows can cause object merging, object shape distortion and even object losses (due to the shadow cast over another object). Miss classification of shadow point as object points may occur in video streams. So, even though the researchers have developed many algorithms which deal with shadows in video streams, the development of efficient techniques for the detection of shadows is still a challenging problem in the area of computer vision. The main concepts utilized for shadow analysis in still and video images are similar, but the purpose behind shadow extraction is somewhat different. Shadow analysis, considered in the context of video data, is typically performed for enhancing the quality of segmentation results. Computer vision (object tracking, segmentation etc.), Image enhancement (developing more pleasing image), Scene re-lighting (changing the light direction) etc. are the main application areas of moving shadow detection processes.

In this paper, the section II describes related works in details. Section III presents a clear idea about the proposed method of shadow detection. In section IV, the pros and cons of the proposed method are given with supports of experimental results. Section V gives the conclusion and future works.

\section{RELATED WORKS}

Shadows may give rise to false segments in image segmentation process. Also shadows may be wrongly detected as objects in object detection algorithms. Many shadow detection methods have been developed for indoor and outdoor environments with different illumination conditions. This section briefly reviews some of the important research works in shadow detection for both Single and Video Images. For the purpose of survey, the existing works are categorized into two classes, namely, Shadow detection in single images and video sequences. 


\subsection{Shadow Detection in Single Image}

Shadow detection in single image is still remains as a challenging problem. Separation of shadow from object area is difficult problem for single images.

\subsubsection{Methods based on the types of shadow}

E. Salvador, A. Cavallaro, and T. Ebrahimi proposed an approach to detect and classify shadows for still images in [2]. They exploit invariant color features to classify cast and self shadows. In the first level, they utilize edge detection followed by a morphological operation to extract object and cast shadow regions. A dark region extraction process is then applied to identify shadow candidates in the segmented region. In the second level, they obtained an edge map which does not contain the edges corresponding to shadow boundaries. The obtained edge map is used, together with the dark region map, to distinguish between self and cast shadows. This can be used to detect shadows in still images. But some constraints are uniform colored object and non structured surfaces.

Subsequently, E. Salvador, A. Cavallaro, and T. Ebrahimi [3] proposed an approach using invariant color features to segment cast shadows in both still and moving images. In the case of videos, motion detector will identify the moving areas and then only on those areas the analysis will be performed. Still, an initial hypothesis is tested to identify candidate shadow regions and a verification stage is then applied based on color invariance and geometric properties. By a comparison with other algorithms they could have high accuracy on object segmentation.

Li Xu, Feihu Qi, and Renjie Jiang [4] proposed a comprehensive method to remove vague and hard shadows from images. Initially they convert the input image to the logarithmic domain. To separate the vague shadows from a single image they used the derivative of the input image. This vague shadow edge detection is based on the assumption that vague shadows varies slowly across an image, but changes in materials are rapid. In the next stage they used color invariants to separate the hard shadow edges from material edges. At last, a Poisson equation is used in reintegration to reconstruct the shadow free image. This shadow removal system is good for real scene images with both vague and hard shadows.

Rosin et al. [5] assumed that shadow is a region with reduced contrast and shadow region is detected using region growing algorithm. But the problem is that region growing algorithm cannot perform accurately in the penumbra part of the shadow.

\subsubsection{Method based on color spaces used}

There are different types of color spaces like RGB, YcbCr, LAB etc. Corina, Peter Jozesef, Zoltan and Laszlo presented an algorithm to automatically detect shadows and remove them from simple consumer images in [A6]. Initially they represent the picture in the $\mathrm{YCbCr}$ color space. They focused on the $\mathrm{Y}$ channel, and computed its histogram. Then computing the full average of the image at $\mathrm{Y}$ channel, and performed sliding window iteration through the image. In order to decide which pixels belong to the shadow, they used two methods. The pixels that have the intensities lower than $60 \%$ of the full average are considered as shadow. The nonshadow point's average is computed for the sliding window. The pixels that have the intensity lower than $70 \%$ of the window's average is considered as part of the shadow. For shadow removal, they introduced two methods: model based shadow removal and additive shadow removal. First one for correction on $\mathrm{Cb}$ and $\mathrm{Cr}$ channels and second for the correction on the y channel. The shadow detection has good results at homogeneous regions, but for more textured regions it could result in false detections.
K.Emily Esther Rani and G. jemilda [7] introduced a preprocessing step using RGB color model to identify the presence of shadows in an image. Once, the presence of shadow is confirmed then the image is converted into shadow invariant image. To detect the shadow boundaries, TAM (Tricolor Attenuation Model) based shadow detection algorithm is introduced. A pre-processing step to segment the original image into sub regions with similar color is also introduced. The algorithm describes the attenuation relationship between shadow and its non-shadow region. This method can automatically detect and extract the shadows from single still images, complex outdoor scenes and this method does not need any user intervention and prior knowledge.

Liu et al. [8] detected shadow using pixel-level information, region-level information, and global-level information in HSV color space. Pixel-level information is extracted using GMM. Local-level information is used in two ways. First, if a pixel gets a sample that is likely to be a shadow, then not only the GMM of that pixel is updated but the GMM of neighbor pixels is also updated. Second, Markov random fields are employed to represent the dependencies among neighbor pixels. For global-level information, statistical feature is exploited for whole scene over several consecutive frames.

Saritha Murali and V K Govindan in [9] proposed a shadow detection and removal method using LAB color space, which is explained in [10]. The color image is converted from RGB to LAB color space. They assumed that the shadow regions are darker and less illuminated than the surroundings. So it is easy to locate them in the $\mathrm{L}$ channel since the $\mathrm{L}$ channel gives lightness information. The B channel values in outdoor images are lesser in shadow areas. Thus combining the values from $\mathrm{L}$ and $\mathrm{B}$ channels, and then pixels with values less than a threshold is identified as shadow pixels. This method works well only for images whose yellow to blue ratio is maintained within a range.

Again Saritha Murali and V K Govindan [11] have proposed an improved method for shadow detection and removal in which also uses LAB color space. The RGB image is initially converted to $\mathrm{LAB}$ image. The sum of mean value of image in $A$ and $B$ channels are calculated. If it is less than a predefined threshold value, then method proposed in [9] can be used. If it is not in that range, then a different method is employed. Pixels with value in the $\mathrm{L}$ channel less than the difference of mean value in $L$ channel and one-third of standard deviation in $\mathrm{L}$ channel are classified as shadow pixels. Other pixels are identified as non-shadow pixels. The shadow detection using this method may identify some non shadow pixels as shadow pixels. For the correct classification of pixels some morphological operations like dilation followed by erosion and area based thresholding were used. Isolated pixels are removed using morphological operation called cleaning.

\subsubsection{Other methods}

Masashi Baba and Naoki Asada proposed a simple Shadow Removal algorithm in [12]. They removed shadows by two stages, shadow detection and color correction. In this approach they detect shadow by a K-means clustering method on color distribution. From these clusters darker cluster is classified as shadow region. They remove the shadows by modify the brightness and color. Finally a smooth filter will be applied to correct the boundary discontinuity between sunshine and shadow regions.

G D Finlayson, S D Hordley, and M S Drew [13] proposed a method to identify and remove the shadow from an RGB image. The shadow edges are located by combined use of original image and illumination invariant image. And then 
edges are set to 0 . Then this edge representation is reintegrated and lightness recovery is done for shadow removal.

Wang, J.M., Y.C. Chung, C.L. Chang, and S.W. Chen [14] proposed an algorithm to remove shadows from a foreground object obtained after subtraction of an image from a background image. In the first step, the foreground region is analyzed to determine if it contains any shadow based on pixel intensity and energy. If a shadow is suspected to exist based on aggregate statistics of bright and dark pixels, the shadow detection step is performed.

\subsection{Methods for moving shadow detection}

In Ref.[15], M. Kilger used a background suppression technique to find the moving objects and moving cast shadows in the scene. For each object, it exploits the information on date, time and heading of the road computed by its system to choose whether to look for vertical or horizontal edges to separate shadows from objects.

In Ref. [16], M. Saad Al-Garni, and A.Adel Abdennour proposed a background subtraction and edge detection technique for detecting moving vehicles. They follow two procedures; initially, extracting the background automatically, then the detection of moving vehicles which depend on edge detection and background subtraction.

Csaba Benedek and Tamas Sziranyi [17] addressed the color modeling problem of shadows in video sequences. They have compared many well known color spaces and illustrated that the performance of shadow detection is very much depend upon the color space which have been used. So, shadow detection performance can be improved by the selection of appropriate color space. They suggested that for real-life videos CIE L*u*v* color space is most efficient.

S. Gupte, O. Masoud, R. Martin, and N. Papanikolopoulos [18], have used only the background subtraction, and then predicting the background. In this approach, background is not separated but detected and updated using next image sequence. Moving camera, changes in intensity and stopped vehicles may leads to miss detection of moving object. This approach cannot be used in the complex background scenes.

In this paper, we have proposed an improved method to detect the moving shadow from a video sequence. We have combined the Background-Foreground separation technique and Shadow detection method.

\section{PROPOSED METHOD}

Shadow detection can be achieved through different methods depending upon the kinds of shadows. Here, we have proposed a method to detect the moving shadow from video sequences. The method involves 4 major steps:

Step 1: Automatic background extraction (Algorithm1)

Step 2: Automatic foreground separation (Algorithm 2)

Step 3: Shadow detection (Algorithm3)

Step 4: Shadow removal

In most of the existing approaches for shadow detection in video images, different environmental changes like intensity changes and camera movements leads to miss detection of shadow.

The proposed method of shadow detection is based on the following assumptions:

1. Strong and single light source

2. Static background and camera

3. Object movement should be towards or against the light source

4. Object motion is parallel to camera position

\subsection{Automatic Background Extraction}

Background extraction can be achieved by the basic subtraction method. Initially input video will be converted to number of frames. These frames could be analyzed to extract the background automatically. Every frame contains the background of the image and the moving object. Since the camera and the background are static, there will not be much difference in backgrounds in each frame.

To extract the background automatically enough number of successive frames must be available for processing. The automatic background extraction starts by processing each frame (images) as in the following Algorithm 1.

\section{Algorithm 1: Automatic background extraction}

Step1: Convert the input video into ' $n$ ' number of frames ( $\mathrm{n}$ is even), $\mathrm{F}_{0}$ to $\mathrm{F}_{\mathrm{n}-1}$

Step2: Select first and last frames and Compare the intensity values and create a new image such that,

$$
\begin{aligned}
\mathrm{B}_{\mathrm{i}}(\mathrm{x}, \mathrm{y})= & \mathrm{F}_{\mathrm{j}}(\mathrm{x}, \mathrm{y}), \text { if } \mathrm{F}_{\mathrm{i}}(\mathrm{x}, \mathrm{y})=\mathrm{F}_{\mathrm{n}-1-\mathrm{i}}(\mathrm{x}, \mathrm{y}), \\
& \text { for all } 0<=\mathrm{i}<=\mathrm{n} / 2-1 ; \\
& =-1, \text { otherwise. }
\end{aligned}
$$

Step3: Get the background as,

$$
\begin{aligned}
& \mathrm{BG}(\mathrm{x}, \mathrm{y})=\mathrm{B}_{0}(\mathrm{x}, \mathrm{y}) \\
& \mathrm{BG}(\mathrm{x}, \mathrm{y})=\operatorname{Max}\left[\mathrm{BG}(\mathrm{x}, \mathrm{y}), \mathrm{B}_{\mathrm{i}}(\mathrm{x}, \mathrm{y})\right], \\
& \text { for all } 1 \leq \mathrm{i} \leq \mathrm{n} / 2-1
\end{aligned}
$$

In (1), BG is the background image. Even though the backgrounds of every frame are same as it is static, small changes in pixel values may occur due to the changes in illumination. These pixel value variations can be avoided by applying a threshold. The use of morphological operations like dilation and erosion help to increase the accuracy of background extraction.

\subsection{Automatic Foreground Extraction}

After extracting the background image, it is easy to separate the foreground image (In this paper, foreground means the moving parts in the video, the object and its shadow) from the original image frames. The reverse operation of background extraction is applied to separate the foreground; that is, the subtraction of the background from original image will give the foreground. The process of foreground separation from the original frame by simple subtraction of background from the original image is illustrated in Algorithm 2.

\section{Algorithm 2: Automatic foreground extraction}

Input: ' $n$ ' number of frames, $F_{0}$ to $F_{n-1}$, background BG

$$
\begin{aligned}
\text { Step 1: } \mathrm{FG}_{\mathrm{i}+1}(\mathrm{x}, \mathrm{y})= & \mathrm{F}_{\mathrm{i}}(\mathrm{x}, \mathrm{y})-\mathrm{BG}(\mathrm{x}, \mathrm{y}), \\
& \text { for all } 0<=\mathrm{i}<=\mathrm{n}-1
\end{aligned}
$$

$\left(\mathrm{FG}_{1}\right.$ to $\mathrm{FG}_{\mathrm{n}}$ are the foreground of each video frames)

\subsection{Shadow Detection}

Now, we have extracted the foreground image. The next step is the detection of shadow. In our foreground image there are only two segments such as the object and its shadow. We can use an edge detection algorithm to get closed regions (segments). Area of the shadow varies drastically from frame to frame. The region which varies in area and almost equal average intensity values may indicate the shadow region. Algorithm 3 shows the basic shadow detection steps used in [19].

\section{Algorithm 3: Basic Shadow detection}

Input: $\mathrm{N}$ foreground images; $\mathrm{FG}_{1}, \mathrm{FG}_{2}, \ldots, \mathrm{FG}_{\mathrm{N}}$ 
$\mathrm{N}=$ Number of frames

$M=$ Number of Segments for each frame

$\mathrm{AI}=$ Average Intensity

Step 1: Apply edge detection on $\mathrm{N}$ foreground images; Let $\mathrm{S}_{\mathrm{ik}}$ is the kth segment of ith foreground image.

$$
\begin{aligned}
& \text { Step 2: for } \mathrm{k}=1 \text { to } \mathrm{M} \\
& \text { if }\left[\operatorname{Area}\left(\mathrm{S}_{\mathrm{ik}}\right) \text { varies for } \mathrm{i}=1 \text { to } \mathrm{N}\right] \\
& \quad \operatorname{AND} \\
& \text { if }\left[\mathrm{AI}\left(\mathrm{S}_{\mathrm{ik}}\right) \text { is almost equal for } \mathrm{i}=1 \text { to } \mathrm{N}\right] \text { then }
\end{aligned}
$$

2.1 Label all segments $S_{i k}$ for $i=1$ to $\mathrm{N}$ as shadows;

2.2 Separate $S_{\text {ik }}$ for each $\mathrm{i}=1$ to $\mathrm{N}$ from the foreground images.

I* step 2 determines whether the kth segment of each Foreground image is a shadow or not; If so remove kth segment from each Foreground image*/

Accuracy of the proposed shadow detection approach is high as the drastic change in the area happens only with shadows and it is easy to distinguish it from the object in the foreground. As per our assumptions the object is moving towards or against the light source, the shadow grows or shrinks drastically as the object moves. Camera is static and parallel to the direction of the object's motion. Because of the light source is single and strong enough to get clear and strong edges, edge detection will give proper closed regions of object and shadow. This leads to higher accuracy in shadow detection.

Even though the shadow detection in Algorithm 3 gives higher accuracy when compared with other existing methods, the running efficiency is questionable. Applying edge detection and segmentation for separating the shadow and object regions in each frame are time consuming processes [19]. In addition, the algorithm in [9] has significant amount of running cost for comparing area of each segments with others, and the comparison of intensity of segments with segments in other frames for shadow identification.

To improve the efficiency in terms of time, a new method of area based shadow detection is proposed in this paper. It modified the Algorithm 3 to avoid the segmentation of whole foreground image by starting the segmentation from the shadow itself. And this method also helps to avoid comparison of segments. This proposed approach of shadow detection is based on the following principles:

The shadow region in the foreground image is at extreme far from the light source. So, if one could find the location of the light source then it is easy to spot pixels in the shadow region. The area of the foregrounds varies from frame to frame as the area of the shadow varies drastically from frame to frame.

Algorithm 4 describes the improved shadow detection method.

\footnotetext{
Algorithm 4: Shadow detection

Input: $\mathrm{N}$ foreground images; $\mathrm{FG}_{1}, \mathrm{FG}_{2}, \ldots, \mathrm{FG}_{\mathrm{N}}$

Declarations:

$\operatorname{loc} 1=(\mathrm{x}, \mathrm{y})$ coordinates of left most pixel of $\mathrm{FG}_{1}$
}

$\mathrm{A} 1=$ area of image in $\mathrm{FG}_{1}$

loc2 $=(x, y)$ coordinates of left most pixel of $\mathrm{FG}_{\mathrm{N}}$

$\mathrm{A} 2=$ area of image in $\mathrm{FG}_{\mathrm{N}}$

Direction $=$ NULL

Light source $=$ NULL

Step 1: If $(\operatorname{loc} 1<\operatorname{loc} 2)$

$$
\text { Direction = RIGHT; }
$$$$
\text { else }
$$

Direction $=$ LEFT

Step 2: If (A1 < A2 \& \& Direction == RIGHT)

$$
\text { Light source }=\text { LEFT }
$$

elseif $(\mathrm{A} 1<\mathrm{A} 2 \quad \& \&$ Direction $==\mathrm{LEFT})$

Light source $=$ RIGHT

elseif $(\mathrm{A} 1>\mathrm{A} 2$ \& \& Direction $==$ RIGHT $)$ else

Light source $=$ RIGHT

Light source $=$ LEFT

Step 3: For $\mathrm{FG}_{1}$ to $\mathrm{FG}_{\mathrm{N}}$ each frame:

if $($ Light source $==$ LEFT $)$

Grouping starts from the right most pixel of foreground

else

Grouping starts from the left most pixel of foreground.

The above algorithm groups neighbouring pixels with similar intensity values and the result is the group of pixels of shadow.

\subsection{Shadow Removal}

Shadow removal is very easy in this model as we have background and shadow free foreground. By super imposing the foreground on the background we will get perfect output. For instance, the foreground $\mathrm{FG}_{1}$ can be superimposed on the background $\mathrm{BG}$ for getting shadow free fame $F_{1}$ and foreground $\mathrm{FG}_{\mathrm{N}}$ can be superimpose on background $\mathrm{BG}$ for getting shadow free $F_{N}$. These resultant frames collectively give the shadow free video.

\section{EXPERIMENTAL RESULTS}

The system was implemented in MATLAB R2012a version 7.14.0.739. The results demonstrate the superior performance of the proposed approach. The Fig. 1 and 2 show the results obtained. In Fig.1, the first row is the various frames in the video sequence. Next row gives the foreground of each frame. In Fig. 2, the first row shows the detected shadow and the shadow free foreground is shown in second row. Third row illustrates the Shadow free output frames. The proposed approach works well for images under the constraint that the object should move towards or against the light source.

In order to specify the performance of proposed algorithm, it is important to use some performance measure to evaluate the algorithm. In this paper, Shadow detection rate $\eta$ and the shadow discrimination rate $\xi$, used in [16], are employed as a quantitative evaluation method. The metric $\eta$ describes how well shadows are detected by the algorithm, while $\xi$ indicates how well the method discriminates shadows from actual foreground objects. These metrics can be expressed as, 


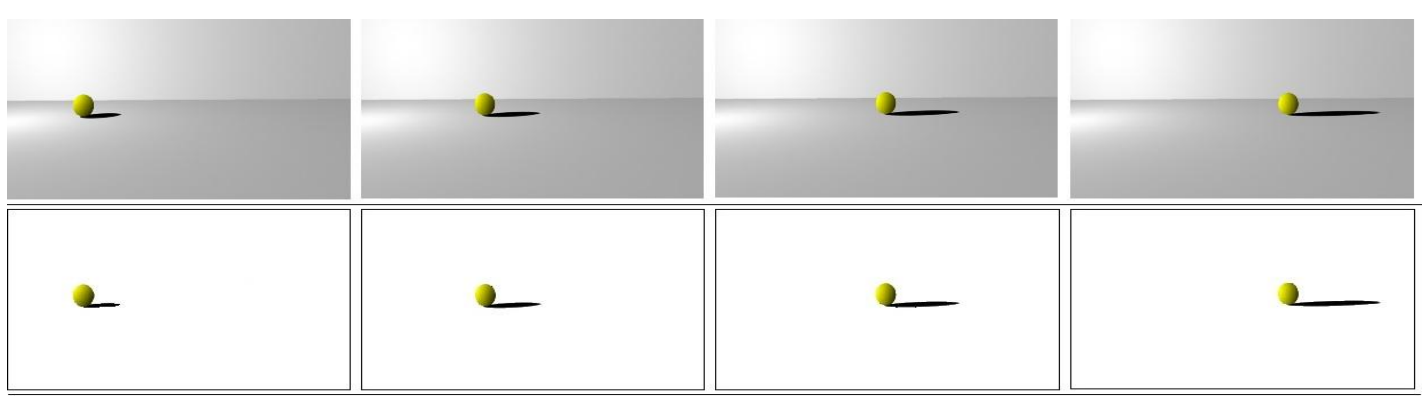

Fig 1: Results of foreground extraction: Four frames of input video (first row); Foreground of each frame with shadow (second row)

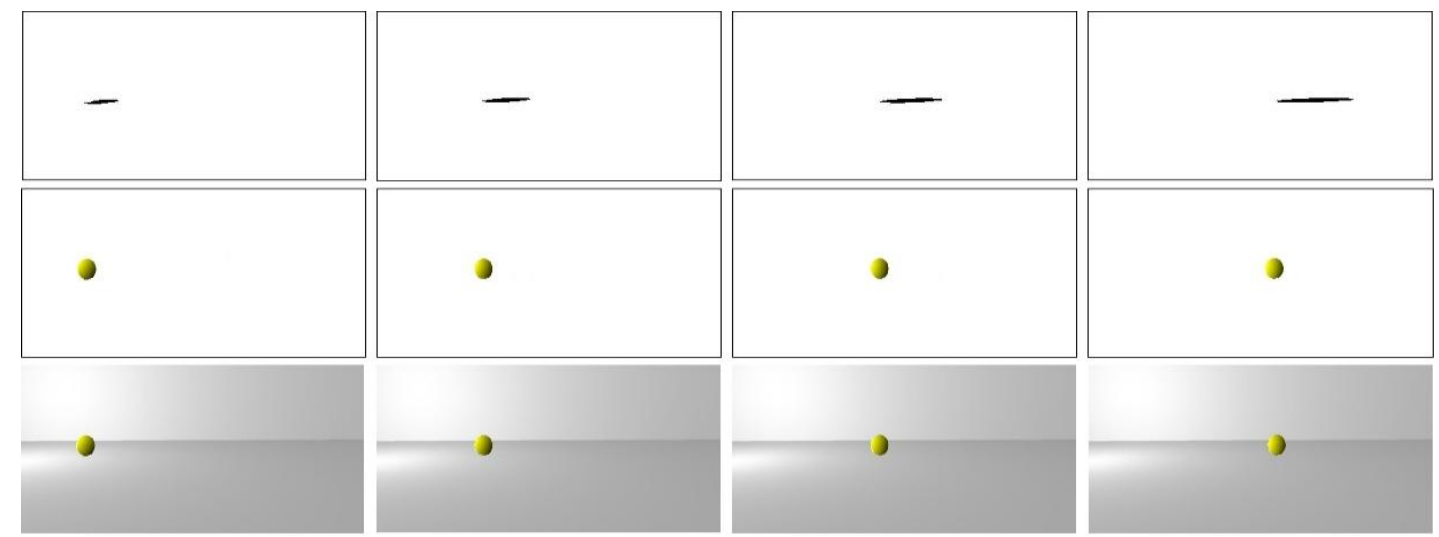

Fig 2: Results of shadow detection algorithm: (row1) Detected shadow; (row2) shadow free foreground; (row3) shadow free output frames

$$
\begin{gathered}
\xi=\frac{T P_{f}}{T P_{f}+F N_{f}} \\
\text { and } \\
\eta=\frac{T P_{s}}{T P_{s}+F N_{s}}
\end{gathered}
$$

In (2), $T P_{f}$ denotes true positive foreground detections (i.e., the number of correctly identified foreground pixels), and $F N_{f}$ denotes false negative foreground detections (i.e., the number of actual foreground pixels that were incorrectly marked as background or shadows). The value $T P_{f}$ is the number of ground-truth foreground pixels that were in fact marked as foreground (i.e., ground-truth pixels minus pixels of the foreground incorrectly marked as shadows). In (3), $T P_{s}$ and $F N_{s}$ denote analogous parameters regarding shadow identification.

Fig. 3 and Fig. 4 shows the results of quantitative analysis of shadow detection and shadow removal. Both the results of the proposed method are compared with some existing methods which are explained in [20]. Also it is compared with the results of algorithm 3 in [19]. In Fig.3, $X$ axis represents frame numbers and $\mathrm{Y}$ axis represents shadow detection rates of five methods including the proposed one. In Fig.4, shadow removal rate of proposed method is compared with other methods.

Table 1 shows the mean values of $\eta$ and $\xi$ for both proposed method and the existing methods. The performance measures obtained for Invariant Color Features (ICF) method given in [3], the shadow suppression algorithm based on edge ratios (ER)[21] , BSSR- method proposed in [20] and Varying Area based Shadow detection method(VASD) in [19] are used for comparison with the proposed method.

Table 1. The mean values of $\eta$ and $\xi$ for different methods

\begin{tabular}{|c|c|c|}
\hline Method & $\begin{array}{c}\text { Mean value of } \\
\eta\end{array}$ & $\begin{array}{c}\text { Mean value of } \\
\boldsymbol{\xi}\end{array}$ \\
\hline ICF [2] & 84.16 & 78.03 \\
\hline ER [17] & 93.10 & 87.62 \\
\hline BSSR [16] & 91.16 & 90.40 \\
\hline VASD & 96.02 & 94.86 \\
\hline Proposed method & 96.28 & 95.38 \\
\hline
\end{tabular}




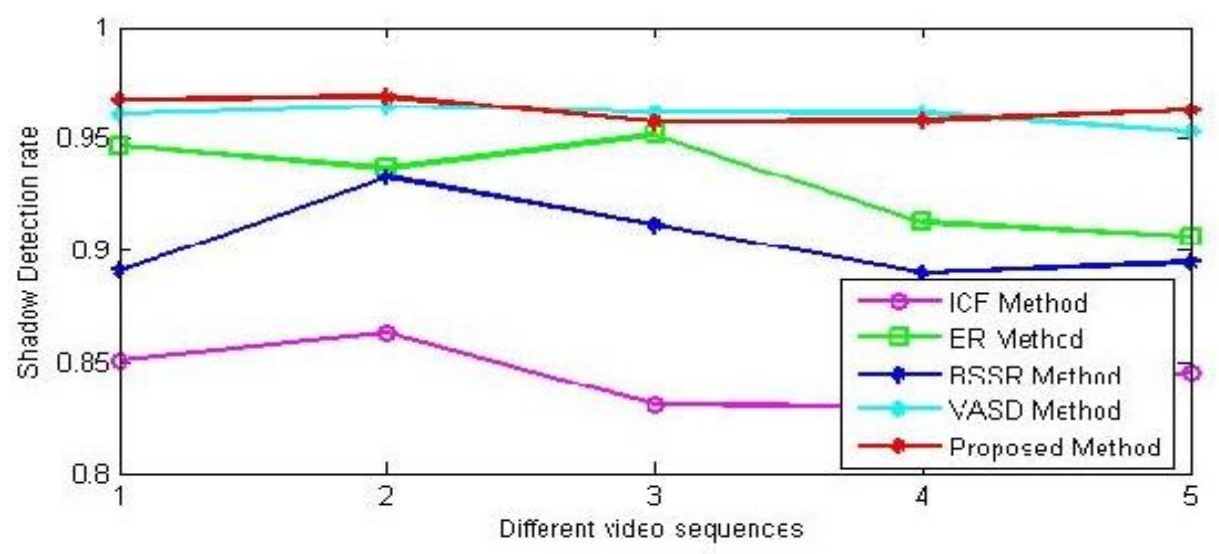

Fig 3: Shadow detection rate of different frames

The results clearly demonstrate the superior performance of the proposed method. When we compare the result of proposed method with VASD method [19], even though there is no much difference, but small improvement in accuracy can be seen.

In order to show the improved efficiency of this Algorithm 4 over Algorithm 3, it is important to compare the executing time of both algorithms for detecting the shadow. Table 2 shows the total time consumption for detecting shadow in all the frames.
From the table it is clear that the average time required for consumption of Algorithm 4 is lesser than the time consumption of Algorithm 3. The difference in time will increase as the number of frames increases. This lesser execution time is accomplished by avoiding overall segmentation and comparisons among segments. In Algorithm 4 , the time used for grouping is proportional to the number of pixels in the shadow region. Grouping is simply achieved by checking neighbor pixels for similar intensity values. Accuracy of this grouping will be high as this process of grouping starts from the shadow region itself. By using more optimizations in implementation the running time can be reduced further.

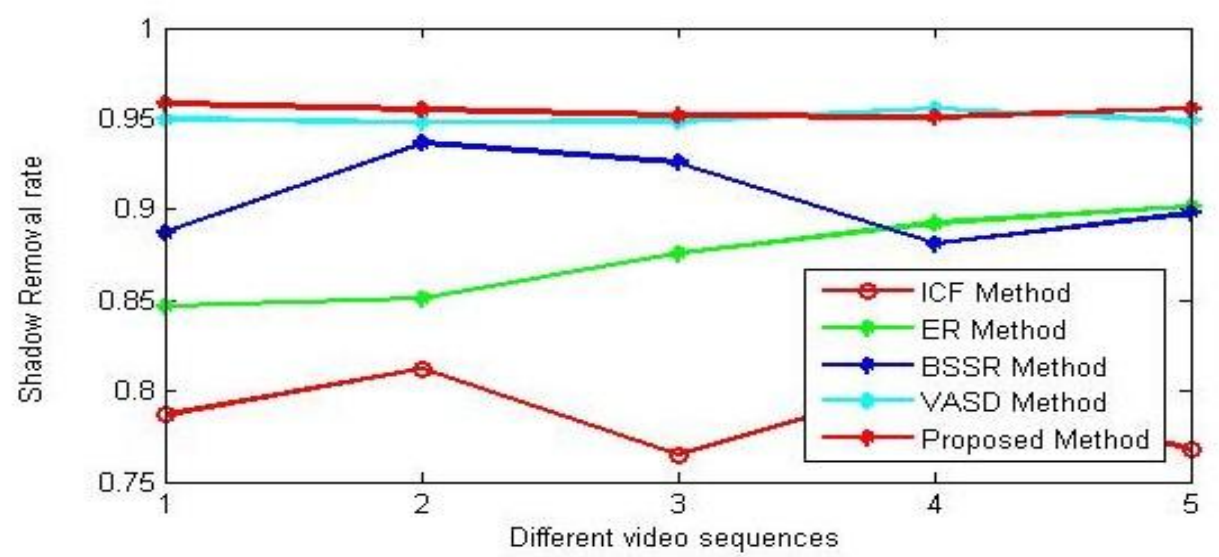

Fig 4: Shadow removal rate of different frames 
Table 2. Comparison of time consumption (Unit: In Sec.)

\begin{tabular}{|c|c|c|}
\hline Method & $\begin{array}{c}\text { Total time } \\
\text { consumption }\end{array}$ & $\begin{array}{c}\text { Average time } \\
\text { consumption } \\
\text { (520 frames) }\end{array}$ \\
\hline VASD Method & 120 & 0.24 \\
\hline $\begin{array}{c}\text { Proposed } \\
\text { Method }\end{array}$ & 80 & 0.15 \\
\hline
\end{tabular}

\section{CONCLUSION}

The shadow detection and removal is an important preprocessing operation for improving performance of computer vision tasks. Different methods for shadow detection and removal have been proposed in the literature. But each of them specifically considers a type of image or shadows. So, these methods have their own merits and demerits. Moving Shadow detection has an important role in computer vision applications including video conference and vehicle tracking. Video is prone to miss detection of the shadows due to its frame to frame quality differences. Most of the existing techniques struggle with these anomalies. In this paper, we have proposed a method suitable for efficient and accurate detection of shadows under the following constraint environment: Strong and single Light source, static background and camera, planar background, object movement should be towards or against to the light source, and object motion is parallel to camera position. The technique makes use of the property that the area of shadows will be changing from frame to frame. Experimental results demonstrate that the method is very efficient in detection and removal of shadows.

\section{REFERENCES}

[1] Habib Ullah, Mohib Ullah, Muhammad Uzair, and Fasih ur Rehman, "Comparative Study: The Evaluation of Shadow Detection Methods"In proceedings of International Journal of Video \& Image Processing and Network Security, IJVIPNS-IJENS, Vol:10, No:02 1.

[2] E. Salvador, A. Cavallaro, and T. Ebrahimi, 2001, "Shadow identification and classification using invariant color models", In Proceedings of the Acoustics, Speech, and Signal Processing, On IEEE International Conference - Volume 03, ICASSP '01, pages 1545-1548, Washington, DC, USA.

[3] E.Salvador, A. Cavallaro and T. Ebrahimi. 2004, "Cast Shadow segmentation using invariant color features", In proceedings of Computer Vision and Image understanding 04, vol 95, Issue 2, pages 238-259.

[4] Li Xu, Feihu Qi, and Renjie Jiang, 2006, "Shadow removal from a single image", Sixth International Conference on Intelligent Systems Design and Applications, 2:10491054.

[5] P. L. Rosin and T. Ellis, 1996, "Image difference threshold strategies and shadow detection," in $6^{\text {th }}$ British Machine Vision Conference, Birmingham, page(s): 347-356.

[6] Corina, Peter Jozesef, Zoltan and Laszlo, 2011, "Shadow detection and removal from a single image", 19th summer school on image processing, SSIP 2011.
[7] K.Emily Esther Rani and G. jemilda,2011, "Shadow detection in single still images using TAM based Multi step Algorithm”, IJCST, Vol. 2, Issue 4, dec2011.

[8]Z. Liu, K. Huang, and T. Tan, "Cast shadow removal combining local and global features," IEEE computer vision and pattern recognition,page(s)1-8, June 2007

[9] Saritha Murali and V.K. Govindan, "Removal of shadows from a single image", In the Proceedings of First International Conference on Futuristic Trends in Computer Science and Engineering, volume - 4, pages 111-114, ICCT 2012.

[10] Tkalcic. M and J. F. Tasic., "Colour spaces: perceptual, historical and applicational background", EUROCON 2003, pages 304 - 308, vol.1, Sept. 2003.

[11] Saritha Murali , "Shadow Detection and Removal from a single image", MTech Project Thesis, NITCalicut, 2011' 12 .

[12] Masashi Baba and Naoki Asada, 2003, "Shadow removal from a real picture", ACM SIGGRAPH 2003 Sketches $1 \&$ Applications, pages:1-1.

[13] G D Finlayson, S D Hordley, and M S Drew, "Removing shadows from images", In proceedings of the $7^{\text {th }}$ European Conference on Computer Vision-Part IV, pages 823-836, London, UK, Springer-Verlag 2002.

[14] Wang, J.M., Y.C. Chung, C.L. Chang, and S.W. Chen,2004, "Shadow Detection and Removal for Traffic Images", IEEE International Conference on Networking, Sensing and Control, Vol. 1, pp. 649-654.

[15] M. Kilger, "A shadow handler in a video-based real-time traffic monitoring system", Proceedings of IEEE Workshop on Applications of Computer Vision, pp. 1118, 1992.

[16] M. Saad Al-Garni, and A.Adel Abdennour, 2008, "Moving Vehicles Detection using Automatic Background Extraction ", World Academy of Science, Engineering and Technology 24.

[17] Csaba Benedek and Tamas Sziranyi, 2007, "Study on Color Space Selection for Detecting Cast Shadows in Video Surveillance", Pazmony Peter Catholic University, Department of Information Technology, Prater utca 50/A, Budapest, Hungary, pages13-17, Budapest, Hungary.

[18] S. Gupte, O. Masoud, R. Martin, and N. Papanikolopoulos, "Detection and classification of vehicles," IEEE Trans. Intelligent Transportation Systems, vol. 3, no. 1, March 2002, pp. 37 - 47.

[19] Jasmin T. Jose and V.K. Govindan, "Varying Area based Shadow detection in video sequences", Accepted for Springer, AIM2013

[20] Jung, Cláudio Rosito,2009, "Efficient background subtraction and shadow removal for monochromatic video sequences", Multimedia, IEEE Transactions on $11.3,571-577$.

[21] W. Zhang, X. Z. Fang, and X. K. Yang, "Moving cast shadows detection using ratio edge", IEEE Trans. Multimedia, vol. 9, no. 6, pp. 1202-1214, Oct. 2007.] 